\title{
Efficient Maintenance Scheduler for Near Optimum Utilization of Oil Tanks
}

\author{
Fawaz S. Al-Anzi and Khaled Al-Zamel \\ Computer Engineering Department, College of Engineering and Petroleum \\ Kuwait University, P.O. Box 5969, Safat, Postal Code 13060, Kuwait
}

\begin{abstract}
Due to the entry of Kuwait into WTO and the expected liberalization of petroleum management, the oil market is to become more competitive. However, the space limitation and public increasing awareness of environmental protection issues and stricter regulation passed by Environmental Public Authority (EPA) in Kuwait make the need for full utilization of oil tanks a prime requirement of a successful oil business. In order to help oil companies to achieve this and maximize revenues by increasing the availability of tanks, an efficient maintenance scheduling is needed. This study introduces a new hybrid evolutionary algorithm and its implantation for solving real-world problem of oil storage tanks. The algorithm incorporates the American Petroleum Institute (API) standard 650 for open inspection procedure to produce a near optimal schedule for maintenance though continuously preserving a population diversity that ensures solution quality and convergence efficiency. The computational results show that the proposed evolutionary algorithm outperforms existing scheduler in literature and produces a higher quality solution which means better revenues though more operational tanks and more environmental protection though maximizing the utilization of existing tanks and less new tanks to build.
\end{abstract}

Key words: Evolutionary, maintenance scheduling, environmental protection, increase revenue, Oil, Kuwait

\section{INTRODUCTION}

In accordance with the entry of Kuwait into WTO entry and the recently issued Petroleum Management Law, the barrier of oil market in Kuwait will been removed. International petroleum vendors can sell their oil products in Kuwait current dominating markets in not so far future. This liberalization results in high competition in the oil market. To keep a cutting-edge position, competitors have to efficiently and effectively control the distribution channel of products, which consists of gas stations, pipelines and storage tanks. Due to the space limitation and the residents' increasing awareness in environmental protection issues and stricter regulation passed by Environmental Public Authority (EPA) in Kuwait, the construction of storage tanks is the toughest obstacle for Kuwaiti and international vendors. In addition, according to the Petroleum Management Law, refining vendors or importers must always maintain reserves of 60 days or 50,000 kiloliters. Therefore, they unavoidably have to optimize the tank uses from the domestic oil companies. See Fig. 1 for an example of oil tank farms in Kuwait.

On the other hand, following the American Petroleum Institute (API) standard 650, storage tanks must be inspected every two years. Depending upon the corrosion degree inside the tanks, a so-called "open inspection" procedure will be conducted every five -to ten years. Each tank will take 60 to 240 days of outage for open inspection based on different capacity and construction type. As a result, a well-devised maintenance schedule of storage tanks will substantially help the leaseholder increase revenue attributed to the availability of tanks but assure the statutory reserves without constructing new tanks.

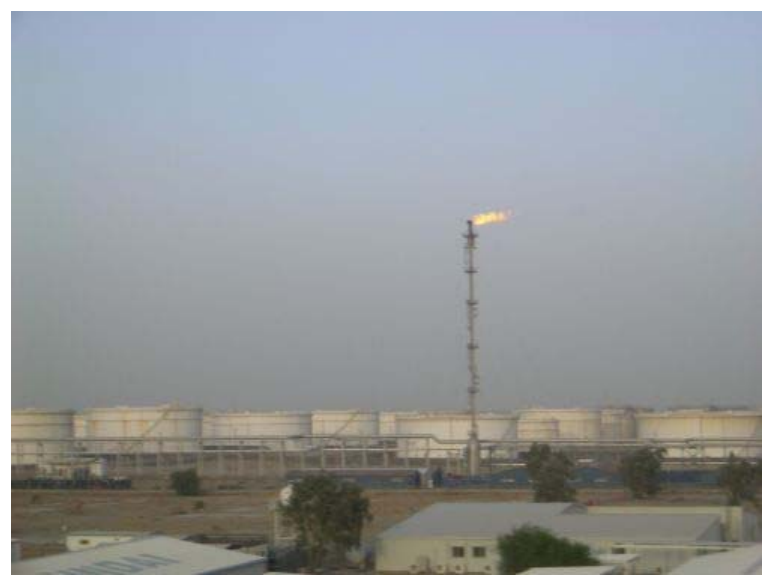

Fig. 1: Oil tank farms in Kuwait

Currently, there is one domestic oil vendor in Kuwait, Kuwait Oil Company (KOC). In the past, the maintenance scheduling relies on the tacit knowledge of senior engineers or the package of linear programming. For a larger number of tanks, the increasing complexity is too high to be managed manually. In the literature,

Corresponding Author: Fawaz S. Al-Anzi, Computer Engineering Department, College of Engineering and Petroleum, Kuwait University, P.O. Box 5969 Safat Postal Code 13060 Kuwait 
genetic algorithms (GA) have been shown able to tackle complicated scheduling problems ${ }^{[1-7]}$. In particular, GA outperforms other heuristic search approaches, such as simulated annealing and tabu search due to the fact (that it is relatively easy to encode in heuristic space and problem space ${ }^{[8]}$. However, GA is subject to suffer the problem of premature convergence, which makes it tend to fall into local optimum. To amend such limitation, we propose to apply the hybrid evolutionary algorithm (heuristic) ${ }^{[9]}$ to deal with this real-world scheduling problem.

Problem statement: The process of petroleum refinement automatically runs 24 hours a day. At first the refined oil is stored in storage tanks and then transported from tanks to gas stations through pipelines or by tank trucks. According to API STD 650, the storage tanks must go through a periodic open inspection. The objective is to find a satisfying maintenance scheduling for the outage caused by open inspection in one year. In the refining system, the outage of storage tanks will affect the stability of oil supply. The level of effect is determined by the net reserve of the oil tank company. The net reserve in certain month $\mathrm{m}$ is defined:

$N_{m}=C-\sum_{i=1}^{T} \eta_{i, m}-\omega_{m}$

$N_{m}$ the obtained net reserve in month $m$,

$C$ : the total capacity of oil Tank Company,

$\eta_{i, m}$ : the capacity of outage of the $i$-th tank in month $m$,

$\mathrm{T}$ : the number of tanks and

$\omega_{m}$ : the forecasting maximum load in month $m$.

In addition, there are two constraints for this maintenance scheduling problem:

* The process of maintenance must begin on the first day of a month and end on the last day of a month. Furthermore, the maintenance should be on schedule and cannot be abandoned.

* The volume of net reserve must be greater than zero at any time. The objective is to keep the net reserve maximum during maintenance.

The period of maintenance scheduling under investigation is one year in this study; that is to say, there will be 12 monthly net reserves in one year. Based on conservative estimation, we determined the lowest net reserves as the fitness of the schedule. i.e.

$\mathrm{Net}=\min \mathrm{N}_{m}=\min \left\{C-\sum_{i=1}^{T} \eta_{i, m}-\omega_{m}, m=1-12\right\}$

\section{THE PROPOSED HEURISTICS}

Simulate Annealing Heuristic (SA): The main idea behind simulated annealing heuristic is to have a large number of iterations where in every iteration of the heuristic there is a single random pair exchange in the sequence. If the exchange improves the objective function then it accepts the exchange and the new sequence is preserved. If the objective function does not improve, then it is only allowed to accept the exchange with some small probability $p$. As the number of iterations increases, the probability $p$ for which the heuristic is allowed to accept an exchange that does not improve the objective function is reduced exponentially. This reduction in the probability usually is expressed as a function of a start temperature that is reduced by a cooling factor to reach a final (freezing) temperature. This technique of reducing the probability of accepting non-improving exchanges has proven to be very useful in escaping local optima's during the course of search for global optima. The following is an algorithmic description of the heuristic.

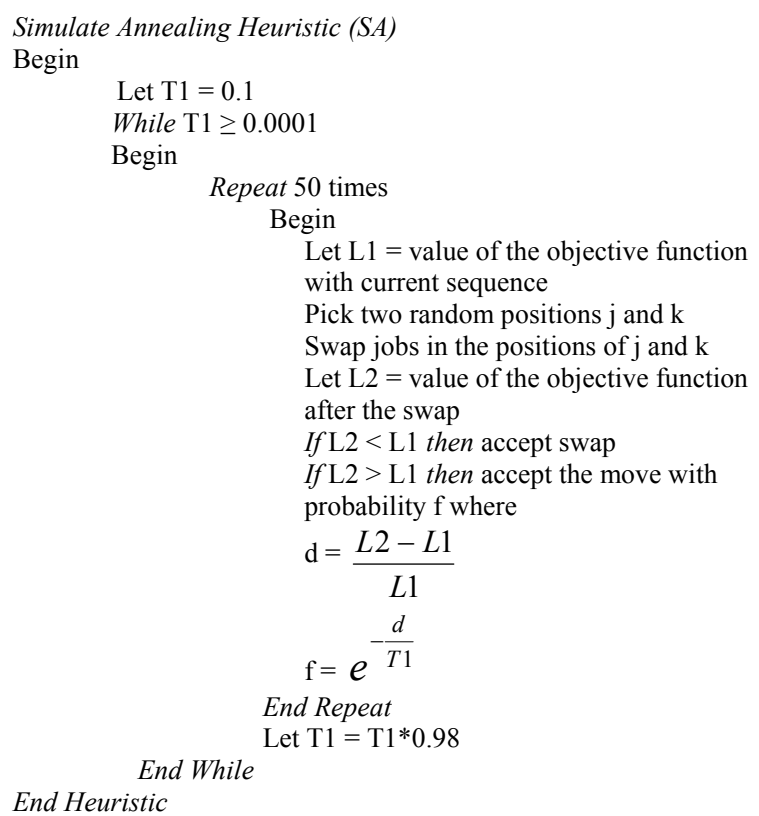

Setting the parameters for the proposed simulated annealing heuristic is essential in achieving a good performance. After some experimentation, the parameters for the simulated annealing heuristic are set as follows; the initial temperature $\mathrm{T} 1$ is set to 0.1 , the cooling factor is set to 0.98 , the final temperature is set to 0.0001 and the number of iterations per fixed temperature is set to 50 .

Regular tabu search heuristic (Tabu): The main idea behind a tabu search heuristic is to have a large number of iterations where in every iteration of the heuristic there is a single random pair exchange in the sequence. The heuristic is only allowed to make an exchange if this exchange improves the objective function and the exchange is not repeating a previous exchange that happened in the previous $h$ iterations which has improved the objective function. The last $\mathrm{h}$ exchanges are kept in a list for checking. This list is called the tabu 
list. This technique has been proven to be very useful for escaping isolation phenomena as well as escaping local optima during the course of search for a global optima. The following is an algorithmic description of the heuristic.

Regular Tabu Search Heuristic (Tabu)

Begin

Initialize Tabu list with maximum size of 4

Select a random sequence as current sequence

Let $\mathrm{T} 1=0.1$

While $\mathrm{T} 1 \geq 0.0001$

Begin

Repeat 50 times

Begin

Let $\mathrm{L} 1=$ value of the objective

function with current sequence

Pick two random positions $\mathrm{j}$ and $\mathrm{k}$

If $(\mathrm{j}, \mathrm{k})$ is not in the Tabu list then

Begin

Swap jobs in the positions of $\mathrm{j}$ and $\mathrm{k}$

Let $\mathrm{L} 2=$ value of the objective

function after the swap

If $\mathrm{L} 2<\mathrm{L} 1$ then

Begin

Add ( $\mathrm{j}, \mathrm{k})$ to front of

Tabu list

If Tabu maximum list

size is exceeded, then

delete the

item at the

Otherwise

end of the list

Reverse and

End If

reject swap

End Repeat

End If

Let $\mathrm{T} 1=\mathrm{T} 1 * 0.98$

End Heuristic

End While

For the proposed tabu search heuristic, setting the parameters is essential in achieving a good performance. After some experimentations, the parameters for the tabu search heuristic are set as follows; the total number of iterations is set to the same value of simulated annealing heuristic (for a fair comparison) and the tabu list size is set to four.

A new tabu search heuristic (Ntabu): The main idea behind the new tabu search heuristic is to introduce the concept of probability of accepting exchanges that do not improve the objective function into the tabu search heuristic. We were inspired to introduce this concept into a tabu search by observing the main concept behind the simulated annealing heuristic. This concept was integrated into the tabu search, which we call a new tabu search heuristic. The new tabu search heuristic is allowed to accept exchanges that are not in the tabu list and as in a regular tabu search, is an exchange that improves the objective function. It is also allowed in the new tabu search heuristic to have exchanges of a second type. The second type is an exchange that is not in the tabu list and does not improve the objective function with a small probability $p$ that is reduced exponentially as the search for the global optima progresses. The following is an algorithmic description of the heuristic.

New Hybrid Tabu Search Heuristic (Ntabu)

Begin

Initialize Tabu list with maximum size of 4

Select a random sequence as current sequence

Let $\mathrm{T} 1=0.1$

While $\mathrm{T} 1 \geq 0.0001$

Begin

Repeat 50 times

Begin

Let $\mathrm{L} 1=$ value of the objective

function with current sequence

Pick two random positions $j$

and $\mathrm{k}$

If $(\mathrm{j}, \mathrm{k})$ is not in the Tabu list then Begin

Swap jobs in the

positions of $\mathrm{j}$ and $\mathrm{k}$

Let L2 = value of the

objective function after the swap

Compute $d$ and $f$ where

$\mathrm{d}=\frac{L 2-L 1}{L 1}$

$\mathrm{f}=e^{-\frac{d}{T 1}}$

If $(\mathrm{L} 2<\mathrm{L} 1)$ or

(L2 $>$ L1 and with

probability f) then

Begin

Add $(\mathrm{j}, \mathrm{k})$ to front of

Tabu list

If Tabu maximum

list size is

exceeded, then

delete the item

at the end of the

list

Otherwise

Reverse and

End If

reject swap

End Repeat

Let $\mathrm{T} 1=\mathrm{T} 1 * 0.98$

End While

End Heuristic

For the proposed new tabu search heuristic, parameters are set to the same values as in simulated annealing and regular tabu search heuristics.

Experiments: According to the practitioner's experience, there is a roughly linear relation between the capacity of storage tank and the needed months for maintenance as shown in Table 1.

In addition, on the basis of the marketing experiences over 50 years, the predicted the maximum loads every month in one year as illustrated in Table 2. 
Table 1: The capacity of tank and the needed months for maintenance

\begin{tabular}{lrrrrrrr}
\hline Tank Capacity & 10 & 20 & 30 & 40 & 50 & 60 & 70 \\
Maintenance (month) & 2 & 3 & 4 & 5 & 6 & 7 & 8 \\
\hline \multicolumn{7}{l}{ Table 2: The maximal loads in a year } \\
\hline Month & 1 & 2 & 3 & 4 & 5 & 6 \\
\hline Maximum Loads & 860 & 850 & 850 & 840 & 830 & 820 \\
Month & 7 & 8 & 9 & 10 & 11 & 12 \\
Maximum Loads & 830 & 820 & 810 & 850 & 830 & 840
\end{tabular}

Table 3: The attributes of 10 tanks

\begin{tabular}{lrrrrr}
\hline Tank Number & 1 & 2 & 3 & 4 & 5 \\
\hline Capacity (kiloliter) & 50 & 70 & 30 & 50 & 50 \\
Maintenance (months) & 6 & 8 & 4 & 6 & 6 \\
Tank number & 6 & 7 & 8 & 9 & 10 \\
Capacity (kiloliter) & 30 & 20 & 10 & 40 & 70 \\
Maintenance (months) & 4 & 3 & 2 & 5 & 8 \\
\hline
\end{tabular}

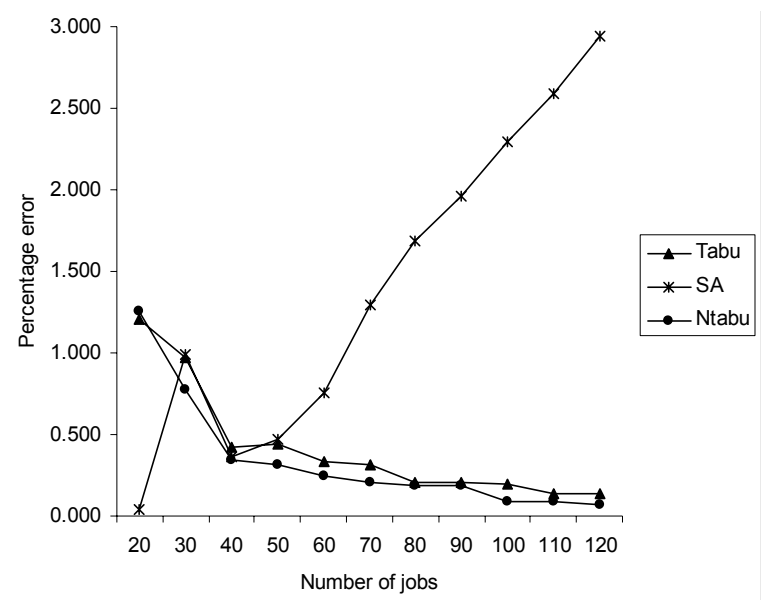

Fig. 2: Error comparison of Tabu, S.A. and Ntabu

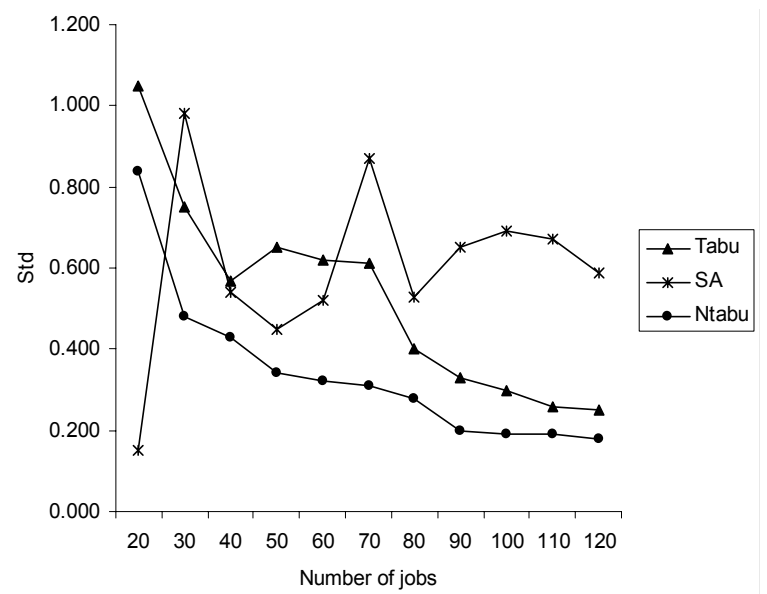

Fig. 3: Std. comparison of Tabu, S.A. and Ntabu

Experimental design: In order to evaluate the performance of proposed approach, we adopt two kinds of data about storage tanks ${ }^{[9]}$. First, we use the practical data of 10 tanks that are arranged to be maintained in certain year according to the dominating petroleum program. The capacities of these tanks and the corresponding month required are shown in Table 3.
Second, the proposed algorithm is experimented on larger scale of problems by simulating 20- and 100 tank cases.

Three sets of data: one of them is the official data (10 tanks) from the dominating program in this year; the others (20 and 100 tanks) are generated randomly to simulate the performance in larger-scale problems.

Performance evaluation: To evaluate the performance of proposed approach, we use the relative error and standard deviation of the compared three heuristics SA, Tabu and Ntabu. Figure 2 and 3 illustrate the superiority of the Ntabu hyped evolutionary algorithm over the regular algorithms.

\section{CONCLUSION}

In this study, we introduced a new hybrid evolutionary algorithm and its implantation for solving real-world problem of oil storage tanks. The algorithm incorporates the American Petroleum Institute (API) standard 650 for open inspection procedure to produce a near optimal schedule for maintenance though continuously preserving a population diversity that ensures solution quality and convergence efficiency. The computational results show that the proposed evolutionary algorithm outperforms existing regular evolutionary algorithms and scheduler in literature and produces a higher quality solution which means better revenues though more operational tanks and more environmental protection though maximizing the utilization of existing tanks and less new tanks to build.

\section{ACKNOWLEDGEMENTS}

The authors would like to thank Kuwait Oil Company (KOC) and Environmental Public Authority (EPA) for their efforts and support in preserving a healthy and safe environment.

\section{REFERENCES}

1. Gen, M. and R. Cheng, 1997. Genetic Algorithms and Engineering Design. A Wiley-Interscience Publication.

2. Goldberg, D.E., 1989. Genetic Algorithm in Search, Optimization and Machine Learning. Addison-Wesley Publishing Company Inc.

3. Levine, D., 1996. Application of a hybrid genetic algorithm to airline crew scheduling. Computers and Oper. Res., 23: 547-588. 
4. Man, K.F., K.S. Tang and S. Kwong, 1999. Genetic Algorithms. Springer-Verlag, London.

5. Negnevitsky, M. and G. Kelareva, 1999. Application of generic algorithms for maintenance scheduling in power systems. Proc. of IEEE Intl. Conf. in Neural Inform. Process., pp: 447-452.

6. Storer, R.H., S.D. Wu and I. Park, 1993. Genetic algorithm in problem space for sequencing problems. In G Fandel, T. Gulledge and A. Jones (Eds), Operations Research in Production Planning and Control: Proc.of a Joint US/German Conf., Springer Verlag, Berlin, Heidelberg, pp: 584-597.
7. Syswerda, G., 1991. Schedule Optimization Using Genetic Algorithms. Handbook of Genetic Algorithms, L. Davis Ed., Van Nostrand Reinhold, N.Y., pp: 332-349.

8. Jain, S. and S. Meeran, 1998. A state-of-art review of job-shop scheduling techniques. Technical report, Department of Applied Physics, Electronic and Mechanical Engineering, University of Dundee, Dundee, Scotland.

9. Ting, C.K., S.T. Li and C.N. Lee, 2001. TGA: a new integrated approach to evolutionary algorithms. IEEE Cong. on Evolutionary Computation, pp: 917-924. 